\title{
LA MUJER EN LA UNIVERSIDAD IDE CASTILLA-LA MANCHA
}

\section{INTRODUCCIÓN}

En la última década la integración escolar y laboral de la mujer se ha incrementado de forma progresiva, pero el logro de una plena e igualitaria participación presenta aún serios obstáculos en cualquier área profesional que analicemos, sobre todo en cuanto se refiere al acceso a puestos de alta responsabilidad.

En este sentido resulta un tanto paradójico el hecho de fijar cuotas, estableciendo un espacio reservado a la mujer para ocupar posiciones de «representación» en el mundo de la política. Este tipo de medidas orientadas a eliminar y corregir las barreras (establecidas por los hombres) que impone la sociedad a la población femenina en realidad, no hacen sino poner en evidencia el trasfondo político subyacente en ellas, y mostrar una preocupación, que no deja de ser superficial, por parte de las instituciones. Preocupación que se solventa con la táctica de aplicar «parches» en lugares estratégicos, en los que la situación

\footnotetext{
* $\mathrm{M}^{\mathrm{a}}$ Adela Ramírez es socióloga. El presente trabajo procede de la ponencia presentada por la autora en el I Encuentro Regional de Sociólogos y Politólogos de Castilla-La Mancha, celebrado en Almagro los días 14 y 15 de junio de 1996.
} 
de desigualdad resulta muy evidente y visible a la luz pública, para con ello presentar ?otra cara? y dar una apariencia de modernidad y progreso democrático, para, a cambio, atraer los votos de un sector muy numeroso de población.

La Universidad española no es ajena a estos hechos, concurriendo además en ella otros factores que contribuyen a esta integración, por un lado el necesario relevo tras la jubilación en masa de la generación de profesorado universitario perteneciente a la posguerra, y por otro la creación de nuevas Universidades, y nuevas facultades, en las ya existentes, que implican en buena lógica un gran número de plazas de nueva creación.

De esta forma, nos encontramos en la actualidad ante una Universidad claramente diferenciada de la de los años 60 ó 70 respecto a la composición de su profesorado, dado que entonces la presencia femenina era prácticamente testimonial.

A esta situación no es ajena la Universidad de Castilla-La Mancha (UCLM), creada en 1985, y por tanto más reciente y permeable a esa participación femenina, como se demuestra en este estudio, donde aparecen una serie de datos coincidentes en líneas generales con las investigaciones realizadas por Fernández, C. (1989) en toda la Universidad española.

En el presente trabajo se estudia la presencia de la mujer en el ámbito de la docencia universitaria en Castilla-La Mancha, tanto a nivel general, como desde diversos aspectos más concretos: la experimentalidad o no de los estudios impartidos, los distintos cuerpos docentes a que pertenecen o su situación laboral desde el punto de vista administrativo (numerarios, interinos o contratados), la edad media de las docentes, así comóel desempeño cargos académicos.

La obtención de estos datos ha sido dificultosa por ser la Universidad una institución reticente a proporcionar cualquier información acerca de su profesorado. Finalmente se ha podido contar con la valiosa colaboración del Centro de Cálculo que nos proporcionó un listado, en el que se reflejaban los siguientes datos:

* Relación nominal del profesorado por orden alfabético

* Fecha de nacimiento

* Cuerpo docente y Centro al que se adscriben 
Para obtener antecedentes sobre la ocupación cargos académicos ha sido necesario recurrir a llamadas telefónicas personales a cada centro. Sobre el dato ?investigación? resultó prácticamente imposible recabar ningún tipo de información fiable, como se explica en el consiguiente apartado, siendo la única fuente que arrojó algo de luz en este sentido, la Memoria de Investigación del periodo 1985-1991, publicada por esta Universidad en 1993.

En el tratamiento de los datos obtenidos se han utilizado las siguientes técnicas:

- Programa Lotus para la confección de la base de datos.

- Paquete estadístico SPSS, principalmente su subprograma Crosstabs para el estudio de la Chi Cuadrado.

\section{ANTECEDENTES Y ORÍGENES DE LA UNIVERSIDAD DE CASTILLA-LA MANCHA}

La Universidad de Castilla-La Mancha nace en 1985 sobre la base de un pequeño núcleo de Escuelas y Colegios Universitarios dependientes hasta entonces de tres Universidades madrileñas (Complutense, Autónoma o Politécnica), para el caso de Ciudad Real, Cuenca y Toledo, o de la Universidad de Murcia para el caso de Albacete, situación un tanto anómala, resultado de las transformaciones ocurridas por la implantación del Estado de las Autonomías y la consiguiente variación de la configuración regional del mapa espa-. ñol, hecho que además influyó notablemente en la organización universitaria.

En el momento de su nacimiento, la Universidad de Castilla-La Mancha contaba con once centros, 402 profesores y 5570 alumnos (Iglesias, A. y Olaya, A. 1993). En el presente curso 1993-94 el número de centros es de 25, 1012 profesores y 23594 alumnos.

Por tanto, podemos afirmar que estamos ante una Universidad de las siguientes características:

A) Marcado carácter multicampus: cinco en la actualidad (Albacete, Almadén, Ciudad Real, Cuenca y Toledo) estando prevista la creación en el próximo curso de otro en Talavera de la Reina , decisión ésta basada más en criterios políticos que de racionalización y preocupación por ofrecer una educación universitaria de calidad. 
B) Repetición de las mismas enseñanzas en distintos campus: así se pueden cursar estudios de derecho en Toledo (Centro Jurídico-Social), Cuenca (Colegio Universitario), Albacete (Facultad de Derecho), existiendo además multiplicidad de campus para otras disciplinas como: Licenciatura en Ciencias Económicas (dos campus), Ingeniería Técnica Agrícola (dos campus), Ingeniería Técnica Industrial (tres campus), Informática (dos campus), Humanidades (dos campus), Ciencias Químicas (dos campus), aparte de las clásicas Escuelas Universitarias que aparecen en todas las provincias como pueden ser las de Profesorado de Educación General Básica y las de Enfermería.

C) Universidad de reciente creación, con todas las ventajas e inconvenientes que ello conlleva.

D) Marcada dependencia de otras Universidades de más solera, para proveerse de profesorado, aunque esta tendencia a depender de otros centros de mayor prestigio es cada vez menor desde la puesta en marcha de los estudios de tercer ciclo en las distintas Facultades, y del aumento de proyectos en materia de investigación.

Como indica Fernández, C. (1989) una de las características de la Universidad española en general es su proximidad a la cúpula del poder político, que trasladada a esta Universidad se transforma en una clara dependencia del poder político regional, que ha llevado en más de una ocasión a revocar decisiones tomadas mayoritariamente en la Juntả de Gobierno de la Universidad. Dicha dependencia se ve incrementada ante las perspectivas de transferencias en materia educativa a la Junta de Comunidades de Castilla-La Mancha, previstas para el año próximo. Por otro lado, la Universidad ha sido la tradicional vía de incursión en la política regional del personal docente universitario, y tampoco es extraño el proceso inverso: el acceso a la docencia desde el mundo político regional. Como puede verse existe un flujo continuo de retroalimentación, que hace que surjan relaciones de interdependencia entre las dos esferas de poder, de gran influencia dentro de nuestro país.

\section{SITUACIÓN DE LA MUJER \\ EN LA UNIVERSIDAD DE CASTILLA-LA MANCHA}

Como ya se ha indicado anteriormente la Universidad de Castilla-La Mancha ha experimentado un espectacular crecimiento en cuanto a profesorado se refiere, pasando de los 402 profesores en 1985 a 1012 en 1993 . Este hecho ha permitido, al igual que en otras 
actividades del país, la incorporación de un buen número de mujeres a la docencia universitaria. En estos momentos, la Universidad de Castilla-La Mancha ocupa el segundo lugar entre las Universidades españolas, con el número más alto de profesorado femenino, por detrás de la Universidad Nacional de Educación a Distancia (Fernández, C., 1989).

Estos resultados observados en su conjunto, ofrecen una visión muy favorable, que hace pensar que, efectivamente los cambios que están teniendo lugar en este terreno, van en una línea progresiva y ascendente, por lo que sólo es cuestión de tiempo, el que la mujer ocupe el lugar que le corresponde en la sociedad. Por tanto, conviene hacer las oportunas matizaciones al respecto y analizar cada una de las variables intervinientes por separado, para lograr unos resultados concluyentes que se ajusten el máximo posible a la realidad.

Como se puede observar en el Cuadro 1, el 33,7\% de los profesores de esta Universidad son mujeres. Sobre el porcentaje total del profesorado de Escuelas Universitarias representan el 34,7\%, similar al 32,9\% de la media estatal, sin embargo el porcentaje de profesoras en el seno de las Facultades $32,6 \%$ sí es claramente superior al de la media estatal que se sitúa en 26,8\% (Fernández, C., 1989).

\section{Distribución del PDI por tipo de centro y sexo}

\section{CUADRO 1 \\ Total de la plantilla del Personal Docenter e Investigador de la Universidad de Castilla-La Mancha}

\begin{tabular}{|l|c|c|c|}
\hline \multirow{2}{*}{ Trpo de Centro } & \multicolumn{2}{|c|}{ Sexo } & Toral \\
\cline { 2 - 4 } & Hombres & Mujeres & \\
\hline Facultades & $329(67,4 \%)$ & $159(32,6 \%)$ & $488(100 \%)$ \\
\hline Escuelas universitarias & $342(65,3 \%)$ & $182(34,7 \%)$ & $524(100 \%)$ \\
\hline Total & $671(66,3 \%)$ & $341(33,7 \%)$ & $1012(100 \%)$ \\
\hline
\end{tabular}

FUENTE: Censo del Centro de Cálculo de la UCLM.

Cuando se agrupan las enseñanzas en experimentales o técnicas (Químicas, Escuelas Técnicas y Enfermería) por un lado y, por otro, las no experimentales (Derecho, Económicas, EGB...) se observa una mayor participación en este segundo grupo, $35,4 \%$ de mujeres frente a un $31,8 \%$ de las experimentales, (Cuadro 2 ). 
Si bien, hay que tener en cuenta que en este grupo figuran los estudios de Enfermería, únicos donde la presencia de la mujer es mayor. Este hecho hace que los resultados se inflen al observar los datos en conjunto. Máxime cuando se da la circunstancia de la total inexistencia de Escuelas Técnicas Superiores, salvo la Escuela de Agrónomos creada el presente curso, que tradicionalmente han sido las que han contado con una menor presencia femenina. En este sentido, esto puede suponer una limitación a la hora de tomar los resultados como definitivos, hasta tanto no concluya la implantación total de centros en esta Universidad.

\section{Distribución del PDI por tipo de enseñanza y sexo}

\section{CUADRO 2}

\section{Distribución del Personal Docente e Investigador según tipo de estudios experimentales/técnicos o no experimentales}

\begin{tabular}{|c|c|c|c|}
\hline Tipo de Enseñanza & & & Total \\
\hline 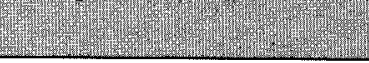 & Hombres & Mujeres & \\
\hline Experimentales o Técnicas & $322(68.2 \%)$ & $150(31,8 \%)$ & $472(100 \%)$ \\
\hline No experimentales & $349(64,6 \%)$ & $191(35,4 \%)$ & $540(100 \%)$ \\
\hline Total & $671(66,3 \%)$ & $341(33,7 \%)$ & $1012(100 \%)$ \\
\hline
\end{tabular}

FUENTE: Censo del Centro de Cálculo de la UCLM.

La explicación detallada de los resultados anteriores podemos contemplarla en el Cuadro 3, donde se han agrupado los distintos tipos de centros.

Por un lado, Facultades de estudios no experimentales y, experimentales y por otro, Escuelas Universitarias de igual categoría, pero sin tener en cuenta la disgregación por Campus. Estos datos confirman y corroboran entre otras cosas las siguientes hipótesis:

- Primero. Predominio de la presencia de la mujer en las Escuelas de Enfermería, que representan un $64,4 \%$, únicos centros en los que el porcentaje se eleva por encima del de hombres, 35,6\%, a continuación le siguen las Escuelas de Formación del Profesorado de EGB con un $41,3 \%$, inferior a la media estatal $(51,3 \%$ ) y las de Relaciones Laborales con un $39 \%$, sin que en ningún caso superen al porcentaje de hombres. 
- Segundo. Le siguen a continuación un grupo de centros cuyos porcentajes se sitúan entre el 30 y 40\%, Químicas 36,6\%, Derecho y Económicas 34,4\% y Letras 33,3\%, para las Facultades son porcentajes en todos los casos superiores a la media estatal, excepto en el caso de las de Letras, cuya media se sitúa por encima del $40 \%$.

- Tercero. En las Escuelas técnicas de grado medio se encuentra uno de los porcentajes más bajos de participación femenina, $19 \%$, pero además hay que tener en cuenta que la mayor parte de las profesoras de estos centros imparten enseñanzas en lo que se denominan asignaturas básicas (Matemáticas, Física, Química, Biología) o Idiomas, mientras que la participación en asignaturas específicas impartidas en cursos superiores es mucho menor.

- Cuarto. La Facultad de Bellas Artes es la que menor número de profesoras presenta $17,1 \%$, inferior al $21,2 \%$ de la media estatal, pudiendo influir el hecho de ser la mayor parte del profesorado asociados residentes en diversas ciudades de España.

\section{Distribución del PDI por grupos de enseñanza afines y sexo}

\section{CUADRO 3 \\ Distribución del profesorado de la UCLM por centros iguales - de similares características de los distintos campus}

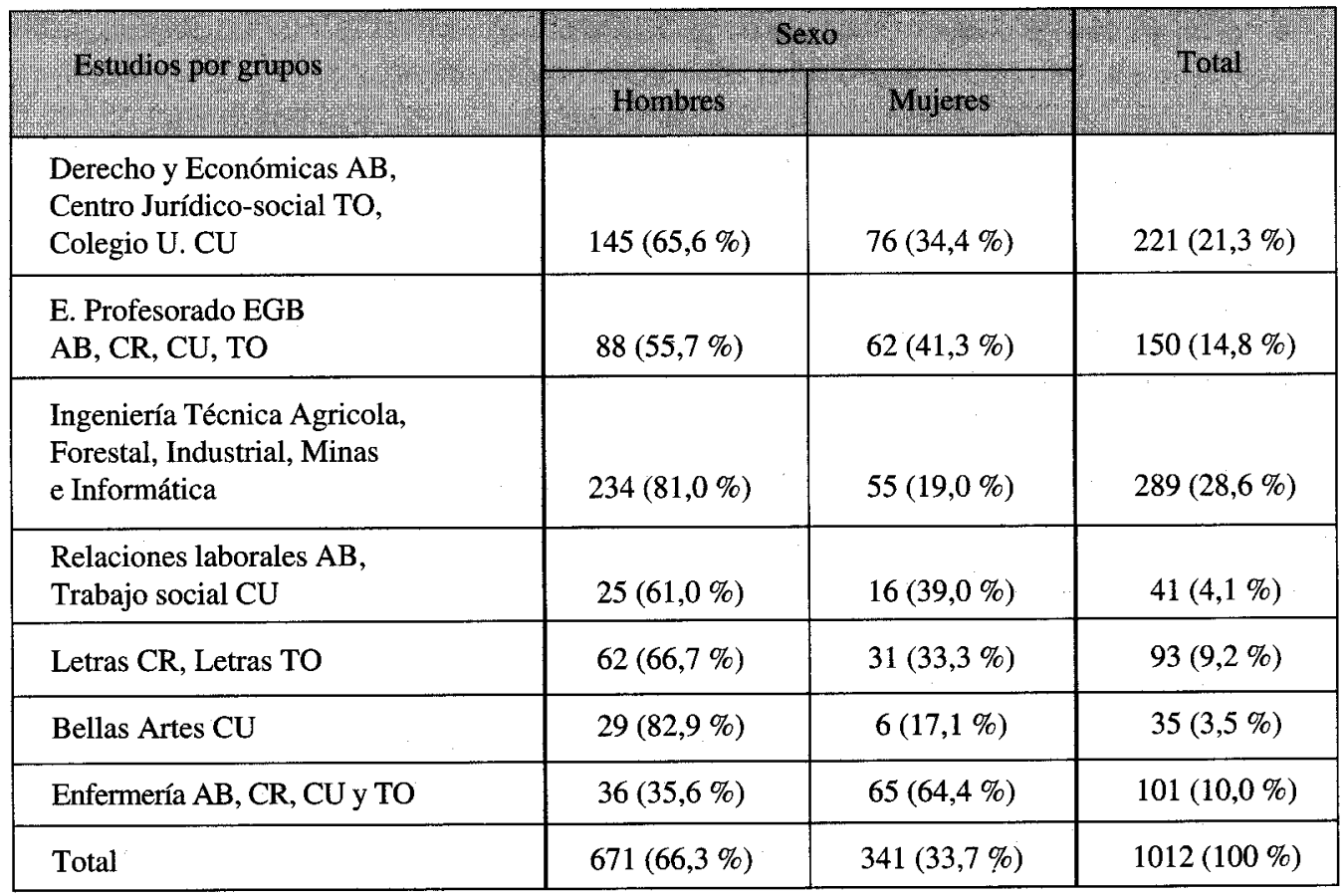

FUENTE: Centro de Cálculo de la UCLM. 


\section{ESTATUS PROFESIONAL ALCANZADO POR LA MUJER}

Se observan claras diferencias entre los distintos niveles alcanzados por cada uno de los sexos, de manera que a medida que se va ascendiendo en la escala de los cuerpos docentes, la participación de la mujer va siendo menor. Esta situación corrobora y muestra las diferencias que se vienen arrastrando del pasado y al mismo tiempo favorece el mantenimiento de una desigual distribución de las esferas de poder, que continúa en manos de los hombres, alcanzando un porcentaje de $88,1 \%$ en el número de Catedráticos de Universidad y de $88,6 \%$ en el de Catedráticos de Escuela Universitaria, mientras que para las mujeres es de $11,9 \%$ y $11,4 \%$ respectivamente (Cuadro 4).

\section{Distribución del PDI por cuerpos docentes y por sexo}

\section{CUADRO 4 \\ Composición de la plantilla del Personal Docente e Investigador de la Universi- dad de Castilla-La Mancha por Cuerpos y sexo en la que aparecen altamente significativas $(p<.001)$}

\begin{tabular}{|l|c|c|c|}
\hline \multirow{2}{*}{ Cuerpolquepertenecen } & \multicolumn{2}{|c|}{ Texo } & Total \\
\cline { 2 - 4 } & Riombres & Mujeres & $42(4,2 \%)$ \\
\hline Catedráticos de Universidad & $37(88,1 \%)$ & $5(11,9 \%)$ & $122(12,1 \%)$ \\
\hline Titulares de Universidad & $78(63,9 \%)$ & $44(36,1 \%)$ & $44(4,3 \%)$ \\
\hline $\begin{array}{l}\text { Catedráticos de Escuela } \\
\text { Universitaria }\end{array}$ & $39(88,6 \%)$ & $5(11,4 \%)$ & $378(37,4 \%)$ \\
\hline $\begin{array}{l}\text { Titulares de Escuela } \\
\text { Universitaria }\end{array}$ & $232(61,4 \%)$ & $146(38,6 \%)$ & $55(5,4 \%)$ \\
\hline Ayudante de Universidad & $27(49,1 \%)$ & $28(50,9 \%)$ & $83(8,2 \%)$ \\
\hline $\begin{array}{l}\text { Ayudante de Escuela } \\
\text { Universitaria }\end{array}$ & $44(53,0 \%)$ & $39(47,0 \%)$ & $155(15,3 \%)$ \\
\hline Asociado Universidad & $117(75,5 \%)$ & $38(24,5 \%)$ & $133(13,1 \%)$ \\
\hline $\begin{array}{l}\text { Asociado de Escuela } \\
\text { Universitaria }\end{array}$ & $97(66,3 \%)$ & $36(27,1 \%)$ & $1012(100 \%)$ \\
\hline Total & $671(66,3 \%)$ & $341(33,7 \%)$ & \\
\hline
\end{tabular}

FUENTE: Censo del Centro de Cálculo de la UCLM. 
Respecto a la situación de Titulares de Universidad y Titulares de Escuela Universitaria los datos no muestran unas diferencias tan claras. El único caso donde la mujer supera al número de hombres es en el nivel de los ayudantes de Universidad, alcanzando un $50,9 \%$, pero en los casos en que ocurre esta circunstancia, podemos comprobar que generalmente las diferencias suelen ser mínimas y además en los niveles más inferiores.

Otro dato muy relacionado con lo expuesto al principio de este apartado, podemos verlo reflejado de nuevo en la distribución de los asociados, al tratarse de profesores que proceden de otras actividades distintas de la enseñanza y donde las diferencias de participación femenina son muy grandes, el porcentaje de profesores Asociados de Universidad alcanza el 75,5\% y el de Asociados de Escuela Universitaria un 73\%, mientras que en las mujeres varían entre un 25 y un $27 \%$, situación que como ya hemos visto se repite en la de los Catedráticos.

No existen sin embargo, grandes diferencias a destacar en cuanto a la situación laboral, como muestra claramente el cuadro 5.

\section{Distribución del PDI por situación laboral y sexo}

\section{CUADRO 5 \\ Situación laboral del PDI de la Plantilla de Personal Docente e Investigador de la Universidad de Castilla-La Mancha, entre los que no se han encontrado diferencias significativas}

\begin{tabular}{|c|c|c|c|}
\hline \multirow{2}{*}{ situaciontiaboral } & & & \multirow{2}{*}{ Total } \\
\hline & & & \\
\hline Funcionario de carrera & $312(66,8 \%)$ & $155(33,2 \%)$ & $467(46,1 \%)$ \\
\hline $\begin{array}{l}\text { Contratado } \\
\text { (Ayudantes, asociados) }\end{array}$ & $282(66,8 \%)$ & $140(33,2 \%)$ & $422(41,7 \%)$ \\
\hline Funcionario interino & $77(62,6 \%)$ & $46(37,4 \%)$ & $123(12,2 \%)$ \\
\hline Total & $671(66,3 \%)$ & $341(33,7 \%)$ & $1012(100 \%)$ \\
\hline
\end{tabular}

FUENTE: Censo del Centro de Cálculo de la UCLM.

Al igual que ocurre en el resto de las Universidades públicas, la edad media del PDI ha descendido en los últimos años, siendo en la de Castilla-La Mancha de 38,65 años para los profesores y de 36,74 años para las profesoras. 


\section{CUADRO 6 \\ Distribución por grupos de edad y sexo del Personal Docente e Investigador de la Universidad de Castilla-La Mancha, entre los que se han encontrado diferencias significativas $(p<.01)$}

\begin{tabular}{|l|c|c|c|}
\hline \multirow{2}{*}{\begin{tabular}{l} 
Grupos de edad \\
\cline { 2 - 4 }
\end{tabular}} & Hombres & Tujeres & Total \\
\hline $20-30$ años & $103(56,6 \%)$ & $79(43,4 \%)$ & $182(19,5 \%)$ \\
\hline $30-40$ años & $274(67,0 \%)$ & $135(33,0 \%)$ & $409(43,8 \%)$ \\
\hline $40-50$ años & $173(68,9 \%)$ & $78(31,1 \%)$ & $251(26,9 \%)$ \\
\hline $50-60$ años & $73(77,9 \%)$ & $15(22,1 \%)$ & $68(7,3 \%)$ \\
\hline+ de 60 años & $17(73,9 \%)$ & $6(26,1 \%)$ & $23(2,5 \%)$ \\
\hline Total & $620(66,5 \%)$ & $331(33,1 \%)$ & $933 *(100 \%)$ \\
\hline Edad media & $38,659,03$ & $36,748,67$ & $38,018,95$ \\
\hline
\end{tabular}

FUENTE: Centro de Cálculo de la UCLM.

* El $n^{\circ}$ total de profesores es inferior al total por desconocerse los datos de edad en algunos casos

\section{POSICIÓN DE LA MUJER EN LAS ESFERAS DE PODER}

Una zona de influencia y toma de decisiones relevantes donde se marcan las pautas generales en cuanto a las actuaciones y líneas que rigen la organización de la Universidad, aparecen claramente delimitadas y distribuidas, entre un conjunto de órganos unipersonales y colegiados, de donde surgen una serie de cargos académicos, unos elegidos por estos órganos y otros simplemente designados.

Es en el reparto de estos cargos académicos tanto electos como designados, donde mayores diferencias aparecen entre hombres y mujeres. Del total de cargos resultantes el 23,8\% lo ocupan mujeres, y este porcentaje disminuye aún más cuando se trata de algunos cargos designados, como en el caso de los vicerrectores donde tan solo aparece una vicerrectora encargada de cuestiones sobre alumnado, cuyas competencias son consideradas de menor responsabilidad que el resto y de menor trascendencia e influencia política, que se extienden más allá del ámbito estrictamente universitario (Cuadro 7). 


\section{Cargos académicos en función del sexo}

\section{CUADRO 7 \\ Cargos académicos que ostenta el Personal docente e Investigador de la Universidad de Castilla-La Mancha distribuidos por sexos.}

\begin{tabular}{|l|c|c|c|}
\hline \multirow{2}{*}{ Cargos académicos } & \multicolumn{2}{|c|}{ Sexo } & Total \\
\hline Rector & Hombres & Mujeres & \\
\hline $\begin{array}{l}\text { Vicerrector ó } \\
\text { Secretario General }\end{array}$ & $1(100 \%)$ & - & $1(0,8 \%)$ \\
\hline Director o Decano Centros & $8(89,9 \%)$ & $1(11,1 \%)$ & $9(7,1 \%)$ \\
\hline $\begin{array}{l}\text { Subdirector vicedecano } \\
\text { Secretario Centros }\end{array}$ & $21(84,0 \%)$ & $4(16,0 \%)$ & $25(19,8 \%)$ \\
\hline Director Departamentos & $42(72,4 \%)$ & $16(27,6 \%)$ & $58(46,0 \%)$ \\
\hline Secretario Departamentos & $18(81,8 \%)$ & $4(18,2 \%)$ & $22(17,5 \%)$ \\
\hline Total & $96(76,2 \%)$ & $30(23,8 \%)$ & $126(100 \%)$ \\
\hline
\end{tabular}

FUENTE: Consulta directa a Centros y Departamentos.

Los porcentajes ascienden cuando se trata de cargos designados en Centros y Departamentos, sobre todo para aquellos más relacionados con tareas burocráticas, consideradas desde siempre ingratas por los hombres, como es el caso de las secretarías de Departamento $(45,55)$ o de Centro $(27,6 \%)$.

En cuanto a los cargos de Decano o Director de centro solamente en aquellos donde son mayoría las profesoras aparecen Directoras, como las Escuelas de Enfermería de Cuenca, Ciudad Real y Toledo, así como la Escuela de Trabajo Social de Cuenca.

Llama también la atención que son varios los centros donde una mujer nunca ha ocupado un cargo académico y una de las razones aparte de los componentes machistas todavía presentes en nuestra sociedad en general y nuestra universidad en particular, puede deberse a que el desempeño de estas actividades va acompañada de una retribución complementaria, por encima de las connotaciones que implica el poder del cargo. Buena muestra de ello la tenemos en tareas asignadas a mujeres, como pueden ser responsables de deportes, actividades culturales, coordinación de planes de estudios etc., que no están retri- 
buidos y donde por regla general el número de ellas es más elevado. Si bien conviene señalar que estos datos son difíciles de confïrmar, pues al no ser oficiales no quedan reflejados de manera formal.

Finalmente, resulta curioso que en el Consejo Social de la Universidad de Castilla-La Mancha, órgano que se supone representa los intereses de la sociedad regional, no participa ni una sola mujer. La razón de ello, podría deberse a que se trata de un órgano más centrado en intereses políticos que académicos o educativos.

Por otra parte es necesario tener en cuenta el hecho de que la mujer dispone de menos tiempo al tener que compaginar las tareas que le exige el desempeño de su profesión con las tareas del hogar y el cuidado de los hijos, incluidos los periodos de embarazos, dado que nuestra sociedad no ha asimilado aún plenamente nuevas pautas de comportamiento en lo que se refiere a establecer un reparto entre las tareas del hogar, que generalmente siguen estando exclusivamente en manos de la mujer y cuando ocurre lo contrario, es considerado por parte de los hombres como una «ayuda extra», prestada de forma esporádica.

Además para promocionarse y ascender a puestos superiores, es requisito imprescindible en el currículum, el haber llevado una labor investigadora que requiere mucho esfuerzo y tiempo para ello, este conjunto de circunstancias colocan a la mujer ya desde un principio, en una posición de desventaja a la que ha de enfrentarse, con doble esfuerzo, si quiere competir con el resto de los compañeros.

\section{LA MUJER Y LA INVESTIGACIÓN EN LA UNIVERSIDAD DE CASTILLA-LA MANCHA}

Dentro de la dificultad general para recabar datos acerca del personal docente en la Universidad, la información sobre la participación de la mujer en el campo de la investigación ha resultado especialmente costosa.

Desde 1989 el Vicerrectorado de Investigación puso en marcha un sistema para recopilar información sobre la actividad investigadora de los profesores de la Universidad de Castilla-La Mancha. El sistema consistente en un disquete a rellenar individualmente por cada profesor contiene todos los apartados que incluyen los currícula y permite de esta forma, por un lado confeccionar la memoria de investigación de la Universidad, y por otro 
aplicando unos baremos predeterminados, valorar mediante la puntuación resultante la actividad investigadora de forma individual, así como por Departamentos, para posteriormente y en función de los mismos repartir los fondos de ayuda a la investigación.

La publicidad de los resultados del primer año levantó muchas suspicacias por lo que dicho Vicerrectorado considera, a partir de entonces, secretos los datos así obtenidos, imposibilitando cualquier tipo de acceso a ellos. De aquel primer listado, el único dato interesante que se puede extraer para el presente trabajo sería el de que la mayor puntuación otorgada entre los docentes de toda la Universidad castellano-manchega, la obtenía, con clara diferencia sobre los demás, una catedrática de la Facultad de Químicas.

Refiriéndose a la situación de las profesoras universitarias dentro del terreno de la investigación indica C. Fernández (1989): «La mujer tiene que mostrar un rendimiento mayor para conseguir el mismo apoyo, tiene que hacer un sobreesfuerzo para demostrar lo que sistemáticamente se pone en duda por el hecho de ser mujer».

Este hecho adquiere, lógicamente, una mayor dimensión teniendo en cuenta que en las universidades españolas los puestos claves para la investigación como puedan ser catedráticos o directores de Departamento, son ocupados mayoritariamente por hombres, que además suelen entender el trabajo «en equipo», de una forma muy particular y propio de una sociedad esclavista en la que se establecen diversas castas de esclavos en función del Cuerpo, situación laboral y por supuesto del sexo.

Así, no es extraño que en estos equipos las labores consideradas más ingratas sean encomendadas a mujeres, no siendo luego repartidas por igual las prebendas.

De esta forma es muy difícil que los currícula sean luego de igual consistencia para todos, pues la dirección de proyectos de investigación, las estancias en el extranjero, la publicación de trabajos etc., tienen sus preferencias, cuando el trabajo realizado ha sido por lo menos el mismo.

En el año 1993 la Universidad de Castilla-La Mancha publica la memoria de investigación correspondiente al periodo 1985-1991, de ella se han extraído una serie de datos cuyos resultados se resumen en el cuadro 8. De todos los datos que figuran en dicha memoria se han escogido aquellos que mejor pueden reflejar la actividad investigadora en los distintos departamentos existentes en la Universidad, como son: 
- Proyectos CICYT o financiados con fondos estatales, así como los proyectos financiados con fondos internacionales. El sujeto que se toma como referencia es el director del proyecto.

- Proyectos financiados con fondos de la administración Autonómica, Local o de la propia Universidad, así como los financiados por la empresa privada. Como en el caso anterior es el/la director/a del proyecto quien nos sirve de referencia.

- Artículos científicos publicados en revistas especializadas de ámbito nacional, en este apartado como en los siguientes la referencia es siempre el primer firmante.

- Artículos científicos publicados en revistas especializadas de ámbito internacional.

- Libros publicados en España.

- Libros publicados en el extranjero.

No se pretende, pues sería imposible, juzgar la calidad de estos trabajos, sobre todo teniendo en cuenta la gran diversidad de áreas de conocimiento, tan sólo una comparación cuantitativa.

De esta forma se observa que la responsabilidad de la mujer en las tareas de investigación, es porcentualmente menor que su presencia en la universidad, debido a las razones esgrimidas anteriormente y sobre todo por su menor presencia en las áreas de poder, esto provoca a su vez, el que realmente no se refleje, de cara al exterior, el trabajo realizado por las mujeres docentes en las tareas de investigación. Este dato es muy importante, dado que la imagen que recibe el profano a través de los medios de comunicación, conferencias, reuniones con la administración, cursos etc., es la de un mundo científico formado casi exclusivamente por hombres expertos donde las mujeres sólo ocupan un plano secundario y auxiliar, en el conjunto de la comunidad universitaria. Lo que por otra parte, afecta realmente la imagen de la mujer y al mismo tiempo refuerza las pautas ya adoptadas por nuestra sociedad, donde no resulta fácil salir del círculo vicioso en que nos encontramos. 


\section{CUADRO 8 \\ Resumen de actividad investigadora del PDI de la Universidad de Castilla-La Mancha}

\begin{tabular}{|l|c|c|c|}
\hline & \multicolumn{2}{|c|}{ Sexo } & Fotal \\
\cline { 2 - 4 } & Hombres & Mujers & \\
\hline $\begin{array}{l}\text { Proyecto CICYT } \\
\text { ó Internacionales }\end{array}$ & $47(82,5 \%)$ & $10(17,5 \%)$ & 57 \\
\hline $\begin{array}{l}\text { Proyectos regionales, } \\
\text { locales, universidad }\end{array}$ & $163(79,9 \%)$ & $41(20,1 \%)$ & 204 \\
\hline Artículos nacionales & $446(79,2 \%)$ & $117(20,8 \%)$ & 563 \\
\hline Artículos internacionales & $67(71,3 \%)$ & $27(28,7 \%)$ & 94 \\
\hline Libros nacionales & $119(86,2 \%)$ & $19(13,8 \%)$ & 138 \\
\hline Libros internacionales & $3(100 \%)$ & 0 & 3 \\
\hline
\end{tabular}

FUENTE: Memoria de investigación de la UCLM, 1985-1991.

\section{CONCLUSIONES}

Como colofón a este trabajo de investigación sobre la situación de la mujer en la Universidad de Castilla-La Mancha, exponemos en breves líneas las siguientes conclusiones:

- Primero. La jubilación de toda una generación de profesores que accedieron a sus plazas en la posguerra española, y por tanto el aumento de vacantes, es una de las causas del incremento del número de mujeres en la docencia universitaria, coincidiendo también con la práctica incorporación femenina a todas las áreas de actividad del país.

- Segundo. Si bien la mujer tiene una mayor facilidad para acceder a la enseñanza universitaria, le siguen siendo vedados los puestos que impliquen poder, apareciendo sólo de forma testimonial, o en algunos casos, para cubrir apariencias, lo cual demuestra que todavía existe cierto grado de desconfianza por parte de los hombres hacia la capacidad de la mujer para hacerse cargo de dichas responsabilidades.

- Tercero. Las condiciones de trabajo y particularmente la promoción, resulta más difícil para la mujer en la Universidad, debiendo resaltar más que el hombre para poder ascender, máxime cuando la posibilidad de promoción pasa por una activa tarea investigadora. 
- Cuarto. Por todo ello, queda aún mucho camino por recorrer hasta alcanzar una integración plena de la mujer en la Universidad, donde solamente cuenta el esfuerzo y dedicación de cada persona en la labor que se ocupa. Este hecho llama la atención, dado el marco contextual donde tiene lugar y debería hacernos reflexionar sobre el cometido de la Universidad española cuya preocupación no ha de ser solamente la de impartir unos conocimientos especializados a los estudiantes, sino además, interesarse por eliminar, en lugar de colaborar a su mantenimiento, trabas y barreras sociales, así como conceder al principio de igualdad de oportunidades el alcance que realmente tiene, para que no se convierta en una mera fórmula «escrita a lápiz» que se puede borrar cuando «convenga». Hoy puede decirse con $\mathrm{A}$. de Miguel, que la Universidad española es una «fábrica de expedir títulos», en la que cualquier otro tipo de preocupaciones sociales o políticas quedan fuera de su cometido.

- Quinto. Por último añadir que dados los tiempos que vivimos, inmersos en una crisis económica a nivel mundial y con un desmantelamiento del Estado del Bienestar, favorecido por una política neoconservadora que va ganando terreno a pasos agigantados, el panorama futuro aparece un tanto sombrío como para imaginar que las cosas vayan a mejorar. Además, en concreto en el caso español, este desmantelamiento del estado de bienestar se produce incluso antes de haberse desarrollado completamente. $Y$ las consecuencias que este hecho conlleva se reflejan en las tasas de paro, mucho mayores que en el resto de Europa, y que afectan principalmente a los sectores más desfavorecidos y marginados de la sociedad: Los jóvenes que buscan su primer empleo y, como no, las mujeres, que son las primeras en acusar los problemas coyunturales del paro estructural que afecta a nuestra sociedad. Por lo que su comportamiento en esta situación consiste en refugiarse dentro del ambiente del hogar y ocuparse de las clásicas labores de «ama de casa», esperando que vuelvan tiempos mejores.

Según Susan Faludi (1991), esta misma situación ocurre y se extiende en el resto de las sociedades. En la última década se ha observado un poderoso contraataque contra los derechos de las mujeres, una reacción que intenta reducir a la nada un puñado de pequeñas victorias duramente ganadas por las mujeres gracias a los movimientos feministas.

Aparece una opinión difundida por distintos medios, prensa, cine, expertos psicólogos, médicos, políticos y también profesores universitarios, afirmando que el feminismo es responsable de hacer desgraciadas a las mujeres. Es una reacción «ideológica» engañosamente «progresista» y orgullosamente retrógrada, que está siendo desplegada por la retórica frenética de los predicadores de la «nueva derecha». Esto muestra la tradicional resistencia y hostilidad de la sociedad norteamericana a los derechos de la mujer. 


\section{BIBLIOGRAFÍA}

- FALUDI, S. (1991): Reacción. La guerra no declarada contra la mujer moderna, Anagrama Barcelona.

- FERNÁNDEZ, C.: «La mujer en la Universidad Española: Docencia, investigación y poder. Datos y aspectos cualitativos». Revista de Educación, 290, (1989):161-171.

- IGLESIAS A. y OLAYA, A.: «Reflexiones en torno a la Universidad de Castilla-La Mancha» Multicampus. Revista de información universitaria $n^{\circ}$ 6, (1993), Rectorado de la UCLM, Ciudad Real.

- UNIVERSIDAD DE CASTILLA-LA MANCHA (1993): Memoria de Investigación 1985-1991. Servicio de Publicaciones de la UCLM, Cuenca.

- UÑA JUÁREZ, O. y BLEDA GARCÍA, J.M.: «La universidad de Castilla-La Mancha, motor de cambios sociales, culturales y económicos», Sociedad y Utopía, 7, (1996): 173-183. 